\title{
NEW DIII-D TOKAMAK PLASMA CONTROL SYSTEM
}

\author{
by \\ G.L. CAMPBELL, J.R. FERRON, E. McKEE, A. NEREM \\ T. SMITH, E.A. LAZARUS, ${ }^{*}$ C.M. GREENFIELD, and R.I. PINSKER
}

This is a preprint of a paper to be presented at the 17th Symposium on Fusion Technology, September 14-18, 1992, Rome, Italy, and to be printed in the Proceedings.

\section{Work supported by \\ U.S. Department of Energy}

Contract No. DE-AC03-89ER51114

*

JET Joint Undertaking, Abingdon, Oxon, OX14 3EA, England.

\section{GENERAL ATOMICS PROJECT 3466 SEPTEMBER 1992}

\section{GENERAL ATOMICS




\title{
NEW DIII-D TOKAMAK PLASMA CONTROL SYSTEM
}

\author{
G.L CAMPBELL, J.R. FERRON, E. MCKEE, A. NEREM, T. SMITH, E.A. LAZARUS, ${ }^{1}$ \\ C.M. GREENFIELD, and R.I. PINSKER \\ General Atomics, P.O. Box 85608, San Diego, California $92186-9784$ \\ 1 Oak Ridge National Laboratory, Oak Ridge, Tennessee
}

\section{INTRODUCTION}

A state-of-the-art plasma control system has been constructed for use on the DIII-D tokamak to provide high speed real time data acquisition and feedback control of DIII-D plasma parameters (Rets. 1-3). This new system has increased the precision to which discharge shape and position parameters can be maintained and has provided the means to rapidly change from one plasma configuration to another. The capability to control the plasma total energy and the ICRF antenna loading resistance has been demonstruted. The speed and accuracy of this digital system will allow control of the current drive and heating systems in order to regulate the current and pressure protiles and divertor power deposition in the DIII-D machine. Use of this system will allow the machine and power supplies to be better protected from undesirable operating regimes. The advanced control system is also suitable for control 'gorithm development fior future machines in these areas and others such as distuption avoidance.

The DIII-D tokamak lacility (Ref. 4)] is operated for the U.S. Department of Energy by General Atomics Company (GA) in San Diego. California. The DIII-D experimental program will increase emphasis on if heating and current drive in the near future and is installing a cryopumped divertor ring during the tall of 1992. To improve the tlexibility of this machine for these experiments, the new shape control system was implemented. This new advanced plasma control system has enhanced the capabilities of the DIII-D machine and provides a data acquisition and control platform that promises to be usetul far beyond its original charter.

\section{OBJECTIVES AND CAPABILITIES}

Since a major upgrade in 1985 the DIII-D plasma control has been accomplished using a custom built analog computation and feedback system (Ref. 5). The analog system provided the flexibility to generate a variety of divertor and limiter plasma configurations but was time consuming to set up and maintain. The system also allowed feedback control for electron density and plasma current. The new advanced plasma control system design is intended to replace this hardwired controller with one that provides more precise control of shape and position, closed loop control of additional plasma parameters, and the added capability for discharge performance optimization. It was also desirable to reduce the need for deductive or trial methods of specifying shape control parameters. The potential for using DIII- $D$ to understand and develop plasma physics based control algorithms for future machines was also considered.

The resulting hardware (Ref. 3) and software (Ref. 2) configuration is ideal for future implementation of advanced control strategies utilizing neural network and fuzzy logic principles. It was designed to provide general applicability to a variety of high speed data acquisition and control problems, including real-time process control and real-ime data reduction. As an example, this system has been used to calculate frequency and toroidal mode number from two Mirnov probes sampled at $500 \mathrm{kHz}$ (Ref. 2). The custom data acquisition interface is suitable for connection to a variety of standard digitizer devices using a simple control intertace.

\section{IMPLEMENTATION}

The need for flexibility and programmability suggested a digital architecture and the use of a small number of high speed processors with outstanding matrix arithmetic capability. VMEbus was chosen based on the availability of hardware and sottware and flexibility for adaptation to the next generation of fusion devices. The real time computer selected is based on the Intel i860 microprocessor. Manufactured by CSP Inc.. the SuperCard- 2 is available with one or two i860 
processors. It excels at matrix arithmetic, achieving 80 million floating point operations per second and 4 () million integer operations per second. It also has a 64 bit wide direct memory access operating at $20 \mathrm{MHz}$ (160 MBytes/second). VME based digital and analog VO is used for control. The high speed data acquisition proved to be the most challenging aspect of the project. Standard CAMAC based transient digitizers familiar to project personnel were selected for converting analog input data. Real time performance is obtained from these digitizers by moving the data to the processor memory at the rate the digitizers are operating. To accomplish this a high speed buffer was needed between the digitizer data buses and the $i 860$ board. This was realized in the design of a first-in-first-out (FIFO) memory board. mounted as a daughterboard on the i860 CPU card (Fig. 1). Use of a FIFO also simplifies multiple real time processor arrangements as the data moves asynchronously and at the digitizing rate to all processors. The digitizer set chosen for the advanced plasma control system was from the TRAQ family manufactured by DSP Technology, Inc. Model 2812 digitizers convert at $100 \mathrm{kHz}$ but the system will support other models at up to $3 \mathrm{MHz}$ sample rate. A CAMAC based digitizer controller card was designed and built to control data flow and to buffer the TRAQ bus data for use with long cables. The connection of the digitizers, digitizer controller, and FIFO board is illustrated in Fig. 2. Only 3.2 microseconds are required to transfer 128 channels of data to the processor memory. Conversion of the data to floating point format requires an additional 3.2 microseconds of processor time.

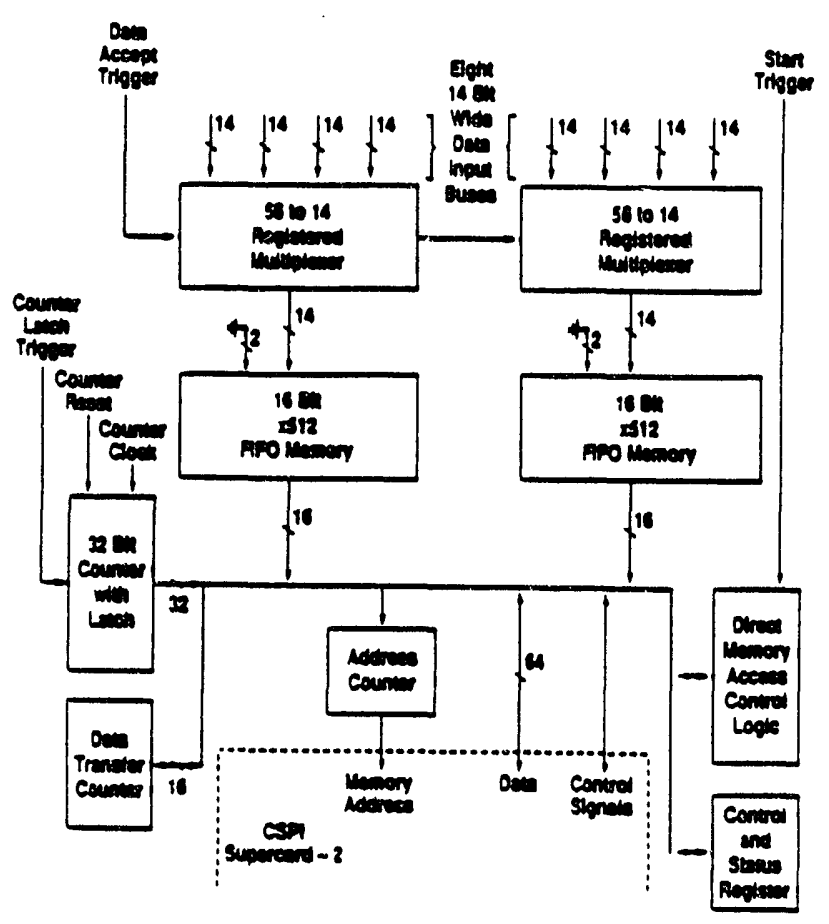

Fig. 1. FIFO board block diagram
For the plasma control application a feedback proportional controller algorithm executes at $10 \mathrm{kHz}$ on the i860 card and is used to set current in the tokamak poloidal coils, controlling discharge shape along with other selected parameters. The control system acquires data from 112 analog signal inputs and provides 18 anilog outputs to control chopper type power supplies which drive the coils. Future extensions will allow control of fueling and current drive sources using additional VMEbus analog and digital VOO.

\section{OPERATING EXPERIENCE}

The advanced plasma control system was made operational on DIII-D during the spring of 1992. It gained rapid acceptance due to ease of use and the ability to quickly make plasma shape changes (Fig. 3). Improvements were noted in the reliability and reproducibility of discharges. An operator interface system was provided in software on the UNIX host processor which is similar to that which had been used for the old analog system. Two unusual experimental plans have used the control system in new ways which would have

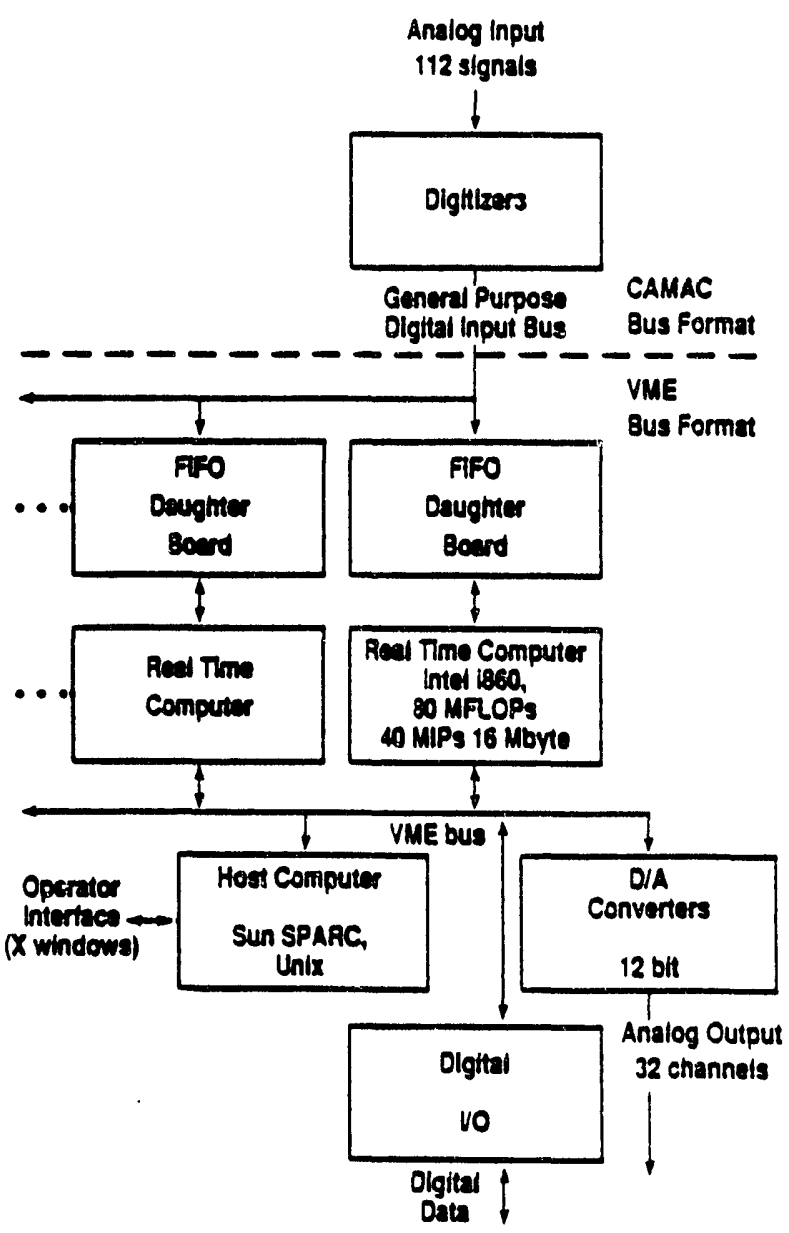

Fig. 2. GA advanced plasma control system 

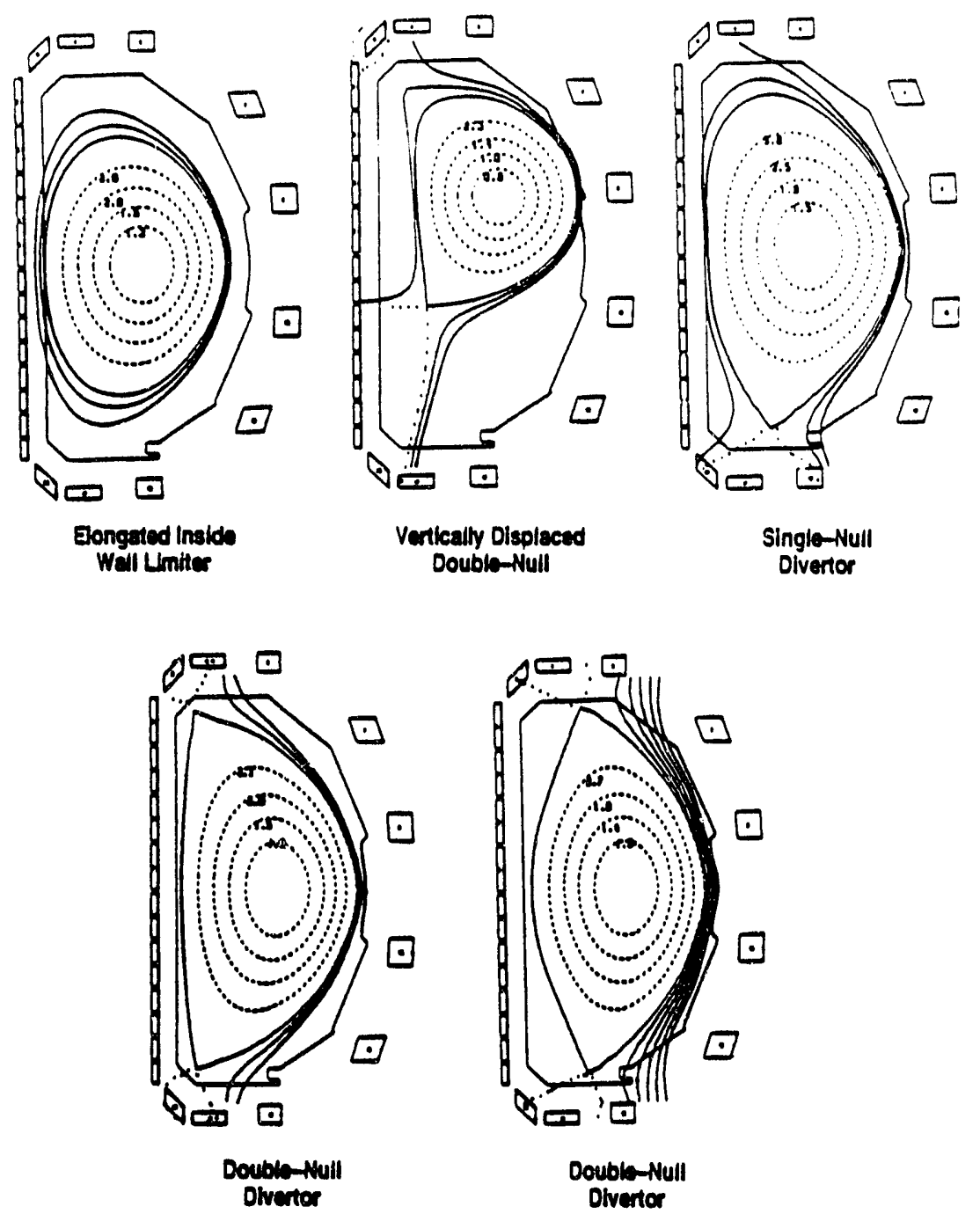

Fig. 3. A wide range of discharge shapes has been produced in DIII-D using the digital plasma control system

been difficult to accomplish before implementation of the new control system. These experiments are briefly described in the following sections.

\section{PLASMA STORED ENERGY CONTROL}

The first experiment was successful in controlling the time evolution of plasma stored energy by pulse width modulating the injected neutral beam power. This is the first time that stored energy control of this type has been accomplished in a large tokamak and the effort resulted in substantially improved plasma performance. The stored energy was inferred from diamagnetic loop data which was used as the feedback quantity. An error signal was derived from a difference between a preprogrammed diamagnetism time evolution and the nieasured quantity. Proportional/differential control was applied to the error. Neutral beam injected power was controlled by turning the accelerator grid voltages on and off with a duty cycle adjusted by the plasma control system during each 25 millisecond period. This varied the average power delivered by four neutral beam sources from zero to $11.3 \mathrm{MW}$.

Figure 4 illustrates the action of the feedback scheme during the course of a discharge. At the beginning of the preprogrammed increase in diamagnetism a large amount of beam power is required (2000 to $2400 \mathrm{~ms}$ ). The beam power is then reduced to a lower level $(2400 \mathrm{~ms})$ and subsequently increases gradually $(2400-3400 \mathrm{~ms})$. As the discharge progresses the stored energy is adversely influenced by the occurrence of edge localized modes (ELMs). These events are indicated by the increase in the photodiode signal [Fig. 4(c)]. At each of the 3 ELM bursts $(2700$ to $3400 \mathrm{~ms}$ ) it can be seen that the control system increased injected power to return the diamagnetism to the programmed level. Aiter $3400 \mathrm{~ms}$ continuous ELMs begin and even the full beam power is insulticient to maintain the requested diamagnetism. 


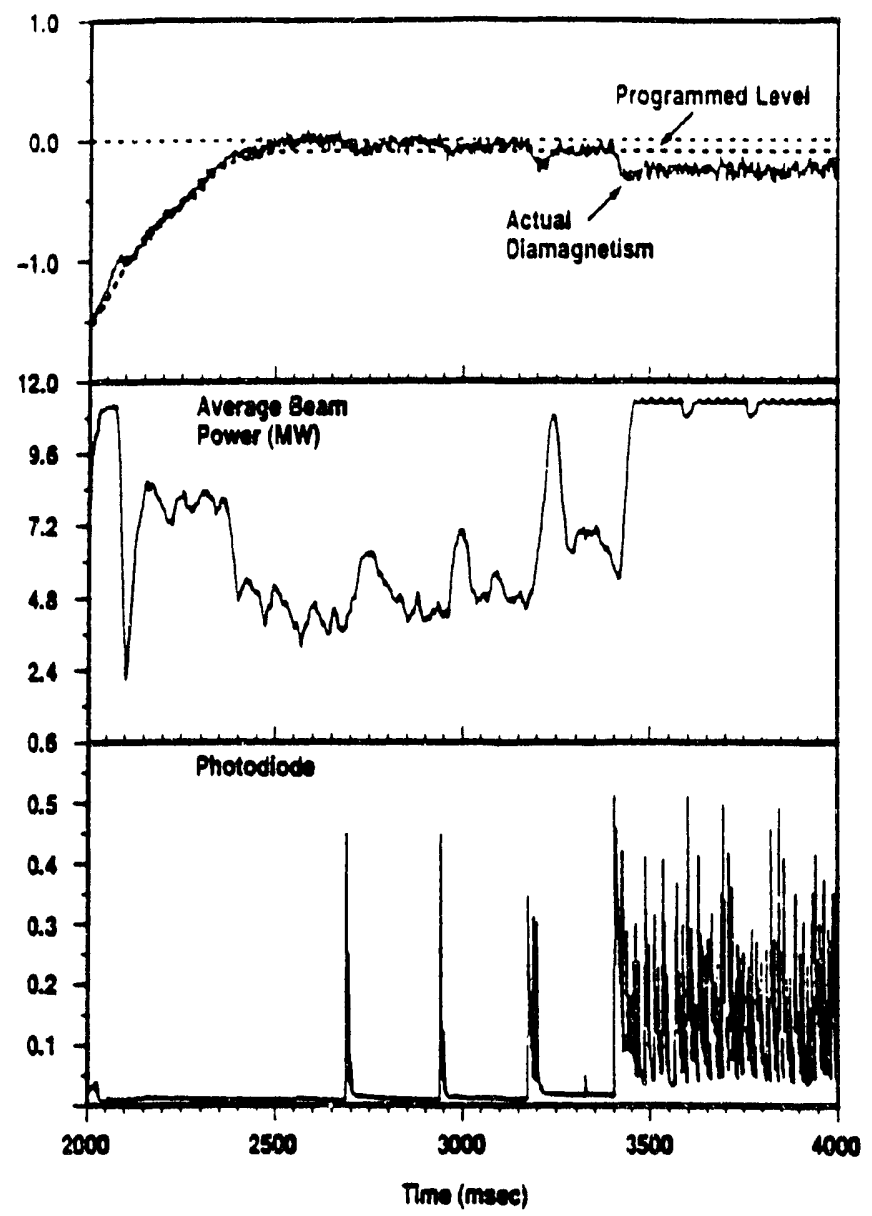

Fig. 4. Controlling plasma stored energy with the advanced control system

\section{ION CYCLOTRON RF COUPLING CONTROL}

The second experiment used the plasma control system to optimize the coupling of Ion Cyclotron (ICRF) waves to the plasma by calculating antenna loading from forward and reflected voltage measurements. These measurements were applied to the proportional differential control algorithm in a similar manner to the first experiment. The control system then adjusted the plasma major radius for constant antenna load.

An example of feedback control of the antenna load resistance is shown in Fig. 5. Figure 5(a) shows the preprogrammed guess for the major radius, the actual major radius of the discharge produced by the feedback system, and a signal that is proportional to the if power. In these experiments it was observed that the gap between the antenna and the plasma required to obtain a given load resistance decreased as the rif power was increased. Thus, Fig. 5(a) shows that as the if power was increased in steps, the plasma major radius also varied in steps. Figure $5(\mathrm{~b})$ is a comparison between the preprogrammed load resistance. essentially a constant, and the load resistance computed by the real time system. The initial spike

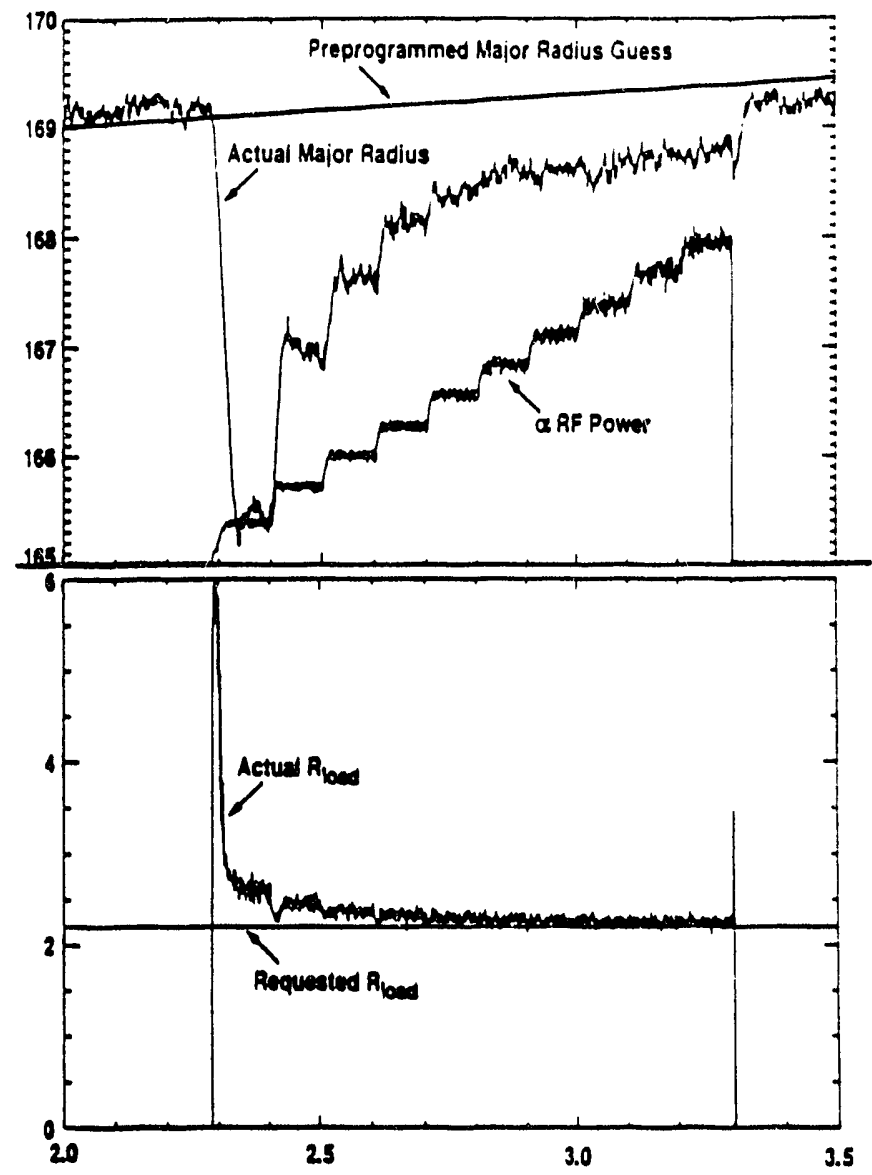

Fig. 5. Controlling ICRF antenna load by charging plasma radius

resulted from the time required to move the discharge after the if was tumed on, but otherwise there is good agreement.

\section{NEAR TERM PLANS}

An engineering project has been undertaken to bring the design and documentation of the advanced control system to a state from which it can be easily reproduced. It will then be used for other data reduction and feedback control applications at DIII-D and elsewhere. During the next operating period at DIII-D the advanced plasma control system will continue to be upgraded in scope. Vertical position control will be addressed for highly elongated plasmas that are close to the ideal stability limit. It will also be possible to change the character of the discharge in mid shot to accommodate a secondary and concurrent experimental plan. Some other possibilities for control extensions include fast wave current drive power and resonance location and electron cyclotron heating power and antenna position. With improvements in machine diagnostics to 
provide real time analysis, current and pressure protile control can be attempted on DIII-D. These diagnostic performance improvements would benetit from the use of a version of the advanced plasma control hardware and software. At this time the GA built fast optical multichannel analyzer (FOMA) is being connected to an advanced control system for real time data analysis and reduction.

\section{CONCLUSION}

The new DIII-D control system has proven a versatile and time saving tool for research. It allows the experimenters to take efficient and full advantage of the capability of the machine and offers the possibility of important investigations into the plasma physics based control algorithms required by the next generation of fusion devices. In addition it has become an attractive choice for implementation of real time diagnostics, data reduction and qualification, and will tind use in high speed process control.

\section{ACKNOWLEDGEMENT}

This work was supported by the U.S. Department of Energy under Contract No. DE-AC03-89ER51114.

\section{REFERENCES}

1. J.R. Ferron, et al., in Proc. of the 14th IEEE/NPS Symposium on Fusion Engineering, (IEEE, New York, 1991) Vol. 2, p. 761.

2. J.R. Ferton, E.J. Strait, Rev. Sci. Instr., to be published in October 1992.

3. J.R. Ferron, Rev. Sci. Instr., to be published in November 1992.

4. J.L. Luxon, et al., Plasma Phys. and Contr. Fusion 32, (1990) 869.

5. S. Kinoshita, et al., General Atomics Report GA-A19584 (1990). 

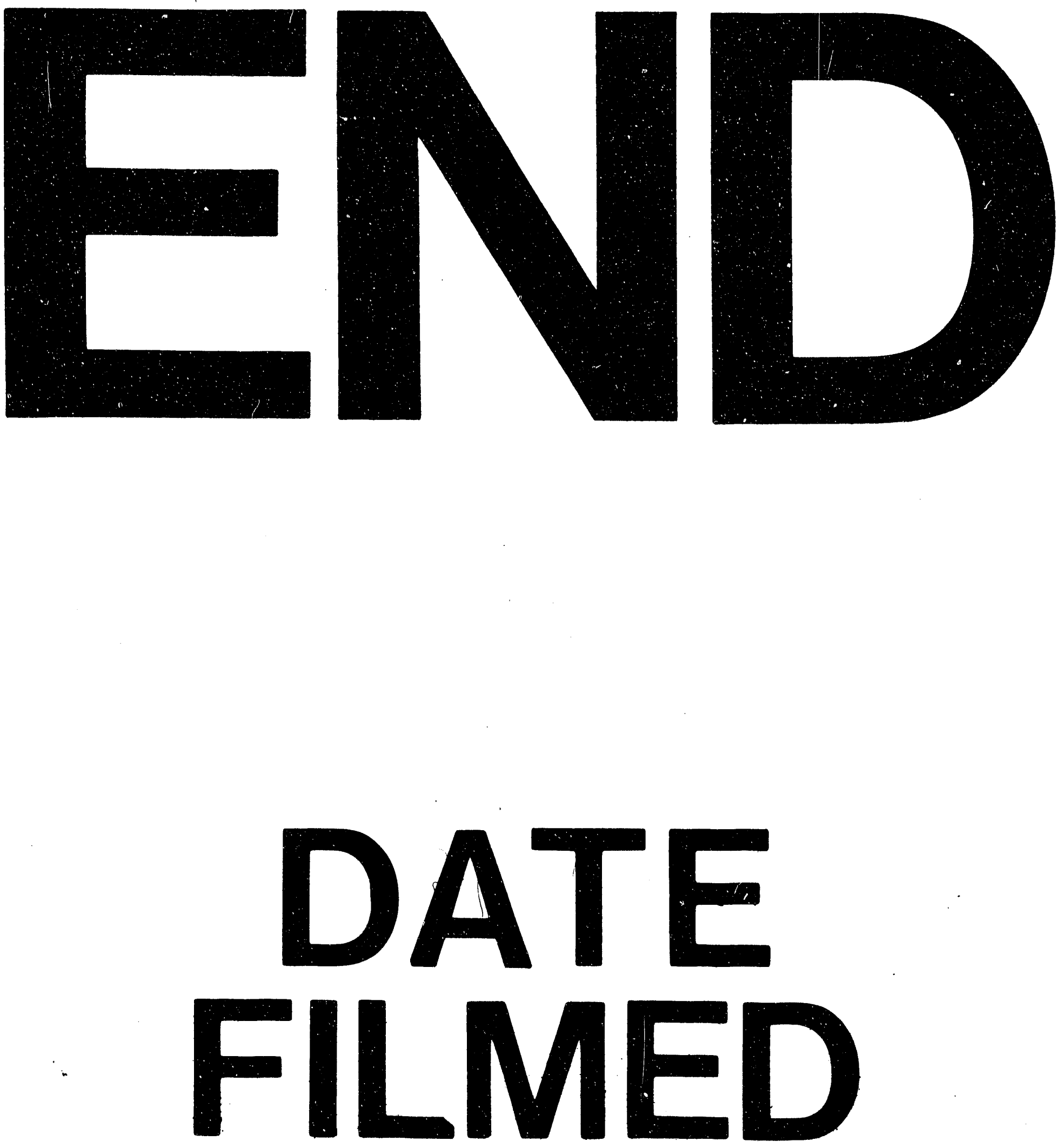

1

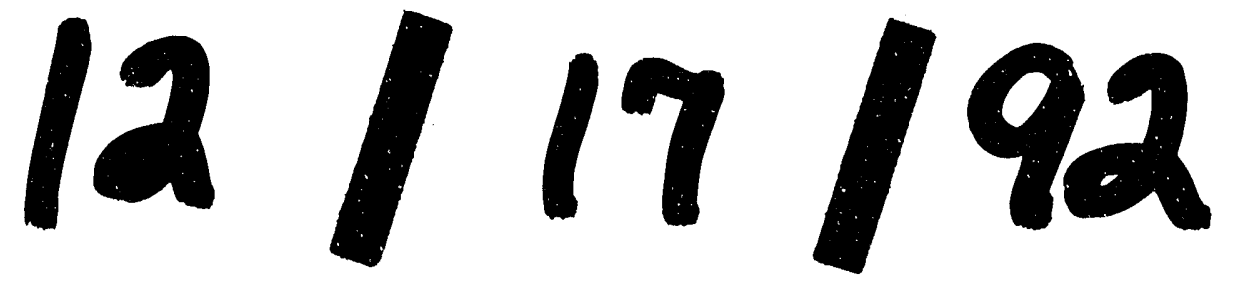


